\title{
Comparação entre SNRI e SSRI na indução da remissão da perturbação depressiva major: uma revisão baseada na evidência
}

Ana Dias Amaral*

\section{RESUMO}

Objetivo: Avaliar a eficácia dos inibidores da recaptação da serotonina e noradrenalina (SNRI) face aos inibidores seletivos da recaptação da serotonina (SSRI) na indução de remissão na perturbação depressiva major.

Fontes de dados: PubMed, bases de dados de medicina baseada na evidência e o Índex de Revistas Médicas Portuguesas.

Métodos da revisão: Pesquisa de normas de orientação clínica (NOC), revisões sistemáticas e meta-análises publicadas até 13/01/2013, bem como de ensaios clínicos (ECA) desde 01/01/2011 até 13/01/2013, utilizando os termos MeSH depressive disorder, major; venlafaxine; duloxetine, bem como os descritores portugueses depressão e antidepressivos de segunda geração. Para classificar a força de recomendação e os níveis de evidência foi utilizada a escala Strength of Recommendation Taxonomy (SORT), da American Family Physician. Resultados: Foram encontrados 223 artigos. Destes, três NOC e nove revisões sistemáticas/meta-análises foram selecionadas. Nenhum ECA cumpria os critérios de inclusão. Tanto os SNRI como os SSRI são considerados terapêutica de primeira linha (nível de evidência [LE] 1) e não há evidência clara de superioridade dos SNRI face aos SSRI (LE 2). Contudo, a evidência mais consistente indica a maior eficácia da venlafaxina sobre a fluoxetina e paroxetina (LE 2). As NOC salientam a importância de escolher um antidepressivo com base no seu perfil de segurança e em aspetos relacionados com o paciente (LE 1).

Conclusões: Tanto os SNRI como os SSRI são uma boa escolha (força de recomendação [SOR] A) para o tratamento da depressão. As características do fármaco e do paciente têm de ser levadas em conta na escolha do antidepressivo (SOR A). Num doente sem morbilidades, alguns SNRI mostram maior eficácia na remissão da depressão, mas não se pode falar de um efeito de classe. A escolha de SNRI em detrimento de um SSRI para atingir a remissão da depressão não é suportada pela evidência atual (SOR B).

Palavras-chave: Depressão; Antidepressivos de Segunda Geração; Indução de Remissão; Medicina Baseada em Evidências.

\section{INTRODUÇÃO}

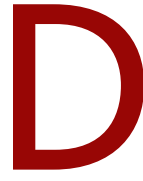
esde o início do projeto Global Burden of Disease da Organização Mundial de Saúde, em 1990, o prognóstico da maioria das doenças passou a ser medido pelos níveis de morbilidade e não tanto pela mortalidade que condicionam. As doenças mentais são, indiscutivelmente, uma das mais relevantes fontes de morbilidade nas populações. De facto, o projeto Global Burden of Disease estima que a perturbação depressiva ma-

*Interna do ano comum, USF Porta do Sol, ULS Matosinhos. jor tornar-se-á a primeira causa de perda de «anos de vida ajustados em função da incapacidade» (disability-adjusted life years) nos países desenvolvidos em $2020{ }^{1}$

Nos últimos meses têm sido veiculadas notícias afirmando que cada vez mais portugueses são diagnosticados com depressão: até um quarto da população adulta portuguesa admite já ter sofrido um episódio depressivo - estudo efetuado por entrevista telefónica e conduzido pela empresa GFK Metris sob orientação científica da Sociedade Portuguesa de Psiquiatria e Saúde Mental. ${ }^{2-3}$ A atual crise económica e as altas taxas de desemprego parecem ser duas causas importantes deste aparente aumento de incidência. 
Entre 1991 e 2002, a prevalência de depressão na população americana mais do que duplicou, atingindo então valores na ordem dos 7\%. ${ }^{4}$ Em Portugal, há poucos estudos visando a evolução da prevalência desta patologia. Um estudo de 2005 mostrou que a prevalência na população geral portuguesa é sobreponível à americana; além disso, 15 a 20\% dos utentes dos Cuidados de Saúde Primários têm diagnóstico de depressão. ${ }^{5}$

De acordo com o Diagnostic and Statistical Manual ofMental Disorders, fourth edition, text revision (DSM-IV-TR), o diagnóstico de perturbação depressiva major é efetuado quando existem sintomas por duas semanas, pelo menos, durante a maior parte do dia, com alteração no estado funcional prévio. São necessários pelo menos cinco sintomas e um deles é obrigatoriamente o humor deprimido e/ou anedonia. Os outros incluem variações no peso corporal de mais de $5 \%$ ou alterações no apetite, perturbação do sono (insónia ou hipersónia), agitação ou lentificação psicomotora, fadiga ou perda de energia, sentimentos de desvalorização pessoal ou culpa excessiva, diminuição da capacidade para tomar decisões ou de concentração e pensamentos recorrentes de morte. ${ }^{6}$

Uma vez que os especialistas em Medicina Geral e Familiar estão cada vez mais envolvidos no tratamento da depressão, estamos interessados em estudar fármacos de primeira-linha usados nesta patologia: inibidores seletivos da recaptação da serotonina (SSRI), como a fluoxetina, paroxetina, sertralina, citalopram e escitalopram, e inibidores da recaptação da serotonina e noradrenalina (SNRI), como a venlafaxina e duloxetina. O principal objetivo deste trabalho foi comparar a eficácia entre os SNRI e os SSRI no que diz respeito à remissão dos sintomas depressivos. Para tal, conduzimos uma revisão baseada na evidência de acordo com as instruções da American Family Physician ${ }^{7}$ e da Revista Portuguesa de Clínica Geral. ${ }^{8}$

\section{MÉTODOS}

Foi efetuada uma pesquisa em bases de dados de reconhecida qualidade na área da Medicina Baseada na Evidência: $\mathrm{Na}$ tional Guideline Clearinghouse, Guidelines Finder, Canadian Medical Association Practice Guidelines InfoBase, The Cochrane Library, Bandolier, DARE, PubMed and TRIP database. O Índex de Revistas Médicas Portuguesas foi também consultado.

Os termos MeSH utilizados foram: depressive disorder, major; venlafaxine; duloxetine. Os descritores portugueses depressão e antidepressivos de segunda geração foram também empregues. Pretendia-se incluir apenas normas de orientação clínica (NOC), revisões sistemáticas, meta-análises e en- saios clínicos aleatorizados (ECA). A pesquisa incluiu todos os artigos encontrados até $13 / 01 / 2013$, exceto no que toca aos ECA (incluídos os estudos entre 01/01/2011 e 13/01/2013).

Os critérios de inclusão desta revisão foram:

- População: adultos (>18 anos), sem outras comorbilidades, no primeiro episódio de depressão major, tratados em regime de ambulatório hospitalar ou nos Cuidados de Saúde Primários;

- Intervenção: SNRI comparados com SSRI;

- Resultados: remissão da depressão entre as 6 e as 12 semanas de tratamento (fase aguda). A remissão foi definida segundo dois critérios: 1) total de até 7-8 pontos na $\mathrm{Ha}$ milton Rating Scale for Depression ou até 10-12 na Montgomery-Åsberg Depression Rating Scale; ou 2) redução em pelo menos $50 \%$ da pontuação reportada em ambas as escalas imediatamente antes da instituição terapêutica. Os critérios de exclusão considerados foram:

- Tipologia dos estudos: estudos não-randomizados; estudos conduzidos em animais;

- População: indivíduos com menos de 18 anos, doentes com história de depressão, depressão concomitante a outras doenças psiquiátricas, depressão associada a fármacos, doentes hospitalizados;

- Intervenção: ausência de comparação com SSRI, comparação entre SNRI, comparação com outras classes de antidepressivos, comparação com psicoterapia, comparação com placebo;

- Resultados: qualquer outro resultado que não o do atingimento da remissão, tal como definida anteriormente. Estudos duplicados ou ECA já incluídos em meta-análises selecionadas foram também excluídos. O nível de evidência e a força de recomendação dos vários artigos, quando ausente, foi atribuído pela autora, de acordo com os critérios da escala Strength of Recommendation Taxonomy (SORT) da American Family Physician. ${ }^{9}$ Classificações baseadas noutras escalas, incluídas em alguns dos estudos, foram convertidas para a escala SORT.

\section{RESULTADOS}

Foram encontrados 223 artigos, 12 dos quais de acordo com os critérios de inclusão: três NOC e nove revisões sistemáticas/meta-análises. Nenhum dos ECA respeitava os critérios pré-estabelecidos. Todos os estudos estão sumarizados nas tabelas I e II.

\section{NOC}

As NOC da Canadian Network for Mood and Anxiety Treat- 


\begin{tabular}{|c|c|c|c|}
\hline NOC & Ano & Conclusão & LE \\
\hline CANMAT $^{10}$ & 2009 & $\begin{array}{l}\text { Basear a escolha em fatores individuais do doente (SOR A). } \\
\text { A venlafaxina é superior à fluoxetina (na remissão). } \\
\text { A duloxetina é superior à paroxetina (na remissão). }\end{array}$ & $\begin{array}{l}1 \\
2\end{array}$ \\
\hline $\mathrm{APA}^{11}$ & 2010 & $\begin{array}{l}\text { Basear a escolha em fatores clínicos ou preferência do } \\
\text { doente (SOR A). } \\
\text { A venlafaxina e duloxetina são tão eficazes quanto os SSRI. } \\
\text { Os SNRI aparentam ligeira superioridade na depressão grave. }\end{array}$ & $\begin{array}{l}1 \\
2\end{array}$ \\
\hline $\begin{array}{l}\text { The Royal College } \\
\text { of Psychiatrists } \\
\text { NICE }^{12}\end{array}$ & 2010 & $\begin{array}{l}\text { Os SSRI devem ser primeira escolha (SOR B). } \\
\text { A venlafaxina e duloxetina não são superiores aos SSRI. }\end{array}$ & 2 \\
\hline
\end{tabular}

Abreviaturas: LE - nível de evidência; SOR - força de recomendação; SSRI - inibidores seletivos da recaptação da serotonina; SNRI - inibidores da recaptação da serotonina e noradrenalina.

\section{Revisões sistemáticas e meta-análises}

Uma revisão da Cochrane de $2012^{13}$ comparou a duloxetina com um SSRI (paroxetina, escitalopram ou fluoxetina). Nenhuma destas comparações se mostrou conclusiva e, no geral, não se encontraram dados estatisticamente significativos que afirmassem ou negassem a superioridade da duloxetina. Os ECA incluídos neste estudo eram similares em termos de desenho de estudo; no entanto, a maioria levantava questões metodológicas - muitos não reportavam de que forma a dupla ocultação (blinding) e a distribuição por grupos (allocation) fora efetuada. Por estes possíveis vieses, foi atribuído a este estudo um nível de evidência 2. Para além disso, muitos eram patrocinados pela indústria farmacêutica com risco de sobrestimação de resultados.

Em 2010, Machado e colegas ${ }^{14}$ compararam os SNRI duloxetina e venlafaxina com vários SSRI (escitalopram, fluoxetina, paroxetina ou sertralina), tendo sido mostrada a superioridade dos SNRI (odds ratio [OR] 1,27, intervalo de confiança [IC] 95\% 1,06-1,52, $p=0,007)$. Os ECA incluídos neste estudo foram classificados com «muito bom» através da lista de verificação de Downs-Black, aplicada pelos próprios autores. Analisados os 27 itens desta escala, verificámos que inclui os cinco critérios de boa qualidade da escala SORT (distribuição aleatória pelos grupos, dupla ocultação, análise por intenção de tratar, tamanho da amostra adequado e seguimento superior a $80 \%$ ). Por este motivo, foi atribuído um nível de evidência 1. Este mesmo investigador ${ }^{15}$ já havia conduzido um estudo semelhante, concluindo que as taxas de remissão eram superiores com SNRI (49,0\%) quando comparadas com SSRI $(37,7 \%)(p<0,001)$. Ao contrário do estudo de 2010, os autores não mencionam neste trabalho se foi efetuada uma análise da qualidade dos ECA incluídos, não sendo possível aferir da sua qualidade metodológica - nível de evidência 2.

As restantes meta-análises estudavam unicamente a venlafaxina. De Silva ${ }^{16}$ comparou este fármaco com o citalopram, escitalopram, fluoxetina, paroxetina e sertralina. A análise do conjunto dos SSRI mostrou que a taxa de remissão era superior com a venlafaxina (OR 1,13, IC 95\% 1,0-1,28, 
TABELA II. Revisões sistemáticas e meta-análises

\begin{tabular}{|c|c|c|c|c|}
\hline Autor, ano & População/intervenção & Resultados & Conclusão & LE \\
\hline $\begin{array}{l}\text { Cipriani et al, } \\
2012^{13}\end{array}$ & $\begin{array}{r}11 \mathrm{ECA} \\
n=3304 \\
\text { Duloxetina vs paroxetina } \\
(6 \mathrm{ECA}), \text { escitalopram } \\
(3 \mathrm{ECA}) \text { ou fluoxetina } \\
(2 \mathrm{ECA})\end{array}$ & $\begin{array}{r}\text { Duloxetina vs paroxetina OR } 1,12 \\
95 \% \text { IC } 0,88-1,43, p=0.36 \\
\text { Duloxetina vs escitalopram OR } 1,31 \\
95 \% \text { IC } 0,87-1,97, p=0,19 \\
\text { Duloxetina vs fluoxetina OR } 0,81 \\
95 \% \text { IC } 0,46-1,44, p=0,47\end{array}$ & $\begin{array}{l}\text { A superioridade da duloxetina não } \\
\text { pode ser afirmada nem negada, } \\
\text { uma vez que as diferenças } \\
\text { encontradas no atingimento de } \\
\text { remissão não são estatisticamente } \\
\text { significativas. }\end{array}$ & 2 \\
\hline $\begin{array}{l}\text { De Silva et al, } \\
2012^{16}\end{array}$ & $\begin{array}{r}26 \text { ECA } \\
n=5858 \\
\text { Venlafaxina vs fluoxetina, } \\
\text { paroxetina, sertralina, } \\
\text { citalopram ou } \\
\text { escitalopram }\end{array}$ & $\begin{array}{r}\text { Venlafaxina vs pool de SSRI OR } 1,13 \\
95 \% \text { IC } 1,0-1,28, p=0,05 \\
\text { Venlafaxina vs fluoxetina OR } 1,16 \\
95 \% \text { IC } 0,99-1,34, P=0,06 \\
\text { Venlafaxina vs paroxetina OR } 1,23 \\
95 \% \text { IC } 0,80-1,87, p=0,35 \\
\text { Venlafaxina vs sertralina OR } 1,28 \\
95 \% \text { IC } 0,71-2,31, p=0,42 \\
\text { Venlafaxina vs citalopram OR } 1,01 \\
95 \% \text { IC } 0,71-1,44, P=0,96 \\
\text { Venlafaxina vs escitalopram OR } 0,92 \\
95 \% \text { IC } 0,62-1,35, p=0,66\end{array}$ & $\begin{array}{l}\text { Embora a análise geral tenha } \\
\text { mostrado uma significância limite } \\
\text { favorecendo a venlafaxina em } \\
\text { detrimento dos SSRI, a análise dos } \\
\text { fármacos individuais não mostrou } \\
\text { superioridade da venlafaxina sobre } \\
\text { qualquer um deles. }\end{array}$ & 1 \\
\hline $\begin{array}{l}\text { Gartlehner et al, } \\
2011^{17}\end{array}$ & $\begin{array}{r}6 \text { ECA } \\
n=1197 \\
\text { Venlafaxina vs fluoxetina }\end{array}$ & OR $1,47,95 \%$ IC $1,16-1,86$ & $\begin{array}{l}\text { A venlafaxina é superior à } \\
\text { fluoxetina no que toca à indução } \\
\text { de remissão. }\end{array}$ & 2 \\
\hline $\begin{array}{l}\text { Machado et al, } \\
2010^{14}\end{array}$ & $\begin{array}{r}15 \text { ECA } \\
n=3094 \\
\text { Venlafaxina ou duloxetina } \\
\text { vs fluoxetina, paroxetina, } \\
\text { sertralina ou escitalopram }\end{array}$ & $\begin{array}{r}\text { SNRI vs SSRI OR } 1,27, \text { IC } 95 \% \\
1,06-1,52, p=0,007\end{array}$ & $\begin{array}{l}\text { Os resultados favorecem o uso de } \\
\text { SNRI sobre os SSRI quando se } \\
\text { pretende atingir a remissão da } \\
\text { depressão. }\end{array}$ & 1 \\
\hline $\begin{array}{l}\text { Bauer et al, } \\
2009^{18}\end{array}$ & $\begin{array}{r}34 \mathrm{ECA} \\
n=7155 \\
\text { Venlafaxina vs fluoxetina, } \\
\text { paroxetina, sertralina, } \\
\text { citalopram ou } \\
\text { escitalopram }\end{array}$ & $\begin{array}{r}\text { Venlafaxina vs pool de SSRI OR 1,19, } \\
\text { IC } 95 \% \text { 1,06-1,34 } \\
\text { Venlafaxina vs fluoxetina OR 1,18, } \\
\text { IC } 95 \% \text { 1,00-1,39 } \\
\text { Venlafaxina vs paroxetina OR 1,49, } \\
\text { IC } 95 \% 1,14-1,96 \\
\text { Venlafaxina vs sertralina OR 1,12, } \\
\text { IC } 95 \% 0,86-1,48 \\
\text { Venlafaxina vs citalopram OR 2,52, } \\
\text { IC } 95 \% 0,60-12,45 \\
\text { Venlafaxina vs escitalopram OR 0,90, } \\
\text { IC } 95 \% 0,61-1,34)\end{array}$ & $\begin{array}{l}\text { Embora a comparação da } \\
\text { venlafaxina com os SSRI como } \\
\text { classe tenha mostrado a } \\
\text { superioridade do primeiro, as } \\
\text { comparações individuais revelaram } \\
\text { que apenas a fluoxetina (no limite) } \\
\text { e a paroxetina são inferiores à } \\
\text { venlafaxina. Nada se pode concluir } \\
\text { no que toca aos restantes três } \\
\text { fármacos. }\end{array}$ & 2 \\
\hline $\begin{array}{l}\text { Llorca et al, } \\
2007^{19}\end{array}$ & $\begin{array}{r}2 \mathrm{ECA} \\
n=483 \\
\text { Escitalopram vs } \\
\text { venlafaxina }\end{array}$ & OR 1,29, IC 95\% 0,84-1,98 & $\begin{array}{l}\text { Embora o estudo aparentemente } \\
\text { favoreça o uso de escitalopram, } \\
\text { não se podem retirar conclusões, } \\
\text { por não existir significado } \\
\text { estatístico. }\end{array}$ & 2 \\
\hline
\end{tabular}




\begin{tabular}{|c|c|c|c|c|}
\hline Autor, ano & População/intervenção & Resultados & Conclusão & LE \\
\hline $\begin{array}{l}\text { Machado et al, } \\
2006^{15}\end{array}$ & $\begin{array}{r}15 \text { ECA } \\
n=2458 \\
\text { SNRI vs SSRI }\end{array}$ & $\begin{array}{r}\text { Taxas de remissão: SNRI 49,0\% } \\
\text { vs SSRI 37,7\% }(p<0,001)\end{array}$ & $\begin{array}{l}\text { Quando se opta por um SNRI, as } \\
\text { taxas de remissão são } \\
\text { significativamente mais elevadas. }\end{array}$ & 2 \\
\hline $\begin{array}{l}\text { Cipriani et al, } \\
2005^{20}\end{array}$ & $\begin{array}{r}9 \text { ECA } \\
n=1891 \\
\text { Venlafaxina vs fluoxetina }\end{array}$ & OR 1,40, IC 95\% 1,15-1,70 & $\begin{array}{l}\text { A venlafaxina levou a taxas de } \\
\text { remissão significativamente mais } \\
\text { altas, quando comparada com a } \\
\text { fluoxetina. }\end{array}$ & 2 \\
\hline $\begin{array}{l}\text { Smith et al, } \\
2002^{21}\end{array}$ & $\begin{array}{r}20 \text { ECA } \\
n=3844 \\
\text { Venlafaxina vs fluoxetina, } \\
\text { paroxetina ou sertralina }\end{array}$ & $\begin{array}{r}\text { Venlafaxina vs pool de SSRI OR 1,43, } \\
\text { IC } 95 \% 1,21-1,71 \\
\text { Venlafaxina vs fluoxetina OR 1,42, } \\
\text { IC } 95 \% 1,17-1,73 \\
\text { Venlafaxina vs paroxetina OR } 1,4, \\
\text { IC } 95 \% 1,05-1,88 \\
\text { Venlafaxina vs sertralina OR } 2,57 \\
\text { IC } 95 \% 1,15-5,82\end{array}$ & $\begin{array}{l}\text { A venlafaxina é significativamente } \\
\text { superior à fluoxetina, paroxetina e } \\
\text { sertralina, quando se analisam as } \\
\text { taxas de remissão. }\end{array}$ & 2 \\
\hline
\end{tabular}

Abreviaturas: LE - nível de evidência; ECA - ensaio clínico aleatorizado; OR - odds ratio; IC - intervalo de confiança; SSRI - inibidores seletivos da recaptação da serotonina; SNRI - inibidores da recaptação da serotonina e noradrenalina; AD - antidepressivos

$p=0,05)$, apesar de não apresentar um significado estatístico conclusivo. Quando se analisaram os cinco antidepressivos individualmente, nenhuma das comparações foi estatisticamente significativa. Todos os ECA foram analisados aquando da sua seleção, quanto à distribuição por grupos, ocultação, seguimento e análise por intenção de tratar, tendo sido incluídos unicamente artigos que preenchessem estes critérios de qualidade. Por estas razões, a meta-análise foi classificada com um nível de evidência 1 .

A meta-análise de antidepressivos de segunda geração de Gartlehner ${ }^{17}$ é extensamente citada e desenvolve numerosas comparações. Uma delas mostra que a venlafaxina é superior à fluoxetina (OR 1,47, IC 95\% 1,16-1,86) quando se pretende estudar a indução de remissão. Analisando os estudos incluídos, verificámos que nem todos cumprem os critérios SORT para serem considerados de boa qualidade. Portanto, atribui-se um nível de evidência 2 a esta meta-análise.

Uma outra meta-análise ${ }^{18}$ comparativa da venlafaxina com SSRI (a maioria dos ECA referindo-se à fluoxetina e paroxetina, mas incluindo também a sertralina, citalopram e escitalopram) concluiu que este SNRI é significativamente mais eficaz na remissão quando comparado com os SSRI (OR 1,19, IC 95\% 1,06-1,34). Não obstante, os fármacos mostraram resultados diferentes individualmente: enquanto a venlafaxina parece superior à fluoxetina, com significado estatístico borderline (OR 1,18, IC 95\% 1,00-1,39) e paroxetina (OR 1,49, IC 95\% 1,14-1,96), comparações com outros SSRI revelaram-se inconclusivas. Alguns dos artigos incluídos são open-label. Devido a esta importante falha na qualidade global da meta-análise, a revisão foi classificada com um nível de evidência 2. Ressalva-se, ainda, o facto de a companhia responsável pela introdução no mercado da venlafaxina ter financiado este estudo.

Llorca ${ }^{19}$ comparou unicamente o escitalopram com a venlafaxina, concluindo que não existem diferenças entre estes antidepressivos. Contudo, não são fornecidas informações quanto à qualidade de ambos os ECA incluídos. Por esta razão, classificou-se esta meta-análise com um nível de evidência 2.

Antes da revisão previamente mencionada, Cipriani ${ }^{20}$ já havia efetuado uma comparação entre a fluoxetina e outros antidepressivos, como a venlafaxina. De acordo com esta meta-análise, a venlafaxina é superior à fluoxetina no que concerne à remissão da depressão (OR 1,40, IC 95\% 1,15-1,70). Os autores declaram que nenhum dos ECA tinha os métodos de randomização apropriadamente descritos; consequentemente, este é um estudo com um nível de evidência 2.

Por fim, uma última revisão ${ }^{21}$ confrontou a venlafaxina e SSRI, nomeadamente a fluoxetina, paroxetina e sertralina. 
No conjunto, a venlafaxina mostrou-se superior aos SSRI (OR 1,43, IC 95\% 1,21-1,71). Para além disso, a venlafaxina foi mais eficaz do que cada um dos três fármacos individualmente. Contudo, os autores afirmam que existiam ECA suficientes apenas para a fluoxetina. Mais, nenhum dos ECA descreveu os métodos de ocultação, pelo que não foi possível aferir da sua adequabilidade. Por estas razões, este estudo tem um nível de evidência 2. Mais uma vez, vários estudos eram patrocinados por companhias farmacêuticas, levantando questões no que toca a possíveis vieses.

\section{CONCLUSÕES}

Muito embora tenham sido pesquisadas múltiplas bases de dados, os estudos neste tópico são escassos e a sua evidência limitada. Em primeiro lugar, existem poucos estudos comparando terapêuticas de primeira-linha na perturbação depressiva major - muitos estudos comparam SSRI ou SNRI com placebo, classes de antidepressivos mais antigas, como os tricíclicos, ou agentes mais recentes, como a mirtazapina. O número de ECA recentes é parco e, embora as metaanálises constituam excelentes sínteses de informação, mais ensaios, envolvendo um maior número de doentes e múltiplos centros, são necessários para clarificar a inconsistência de alguns resultados. Além disso, um número significativo de ECA é conduzido pelos próprios produtores dos antidepressivos, incorrendo em possíveis vieses.

A heterogeneidade dos estudos e contradição de resultados não nos permite afirmar a superioridade dos SNRI face aos SSRI. Aliás, o conceito de superioridade de classe é discutível, uma vez que existe uma considerável variedade farmacológica dentro de cada uma.

Adicionalmente, embora a tolerabilidade e os efeitos laterais estejam além do âmbito desta revisão, não é razoável ignorar aspetos que desempenham um papel tão importante na escolha do antidepressivo ideal para o nosso doente e que, num significativo número de casos, tem outras patologias médicas que não podemos desprezar.

Pesando todos estes aspetos, retiram-se algumas conclusões:

- Aquando da escolha de um antidepressivo, deve considerar-se a tolerabilidade e perfil de efeitos adversos, segurança e custo, bem como aspetos relacionados com o doente - comorbilidades e medicação habitual (SOR A).

- No que toca à indução de remissão de sintomas na fase aguda do tratamento (até às doze semanas), tanto os SSRI como os SNRI são excelentes opções terapêuticas de primeira-linha na perturbação depressiva major (SOR A).
- No que toca a classes de antidepressivos, a escolha de um SNRI em detrimento de um SSRI não é suportada pela evidência atual (SOR B).

- Para pacientes sem outras comorbilidades, a venlafaxina pode ser preferida sobre a fluoxetina ou a paroxetina, considerando-se a indução de remissão na depressão (SOR B).

- Considerando a indução de remissão da sintomatologia depressiva, não existe evidência conclusiva que suporte preferir ou preterir a duloxetina face a qualquer SSRI (SOR B).

\section{AGRADECIMENTOS}

A autora agradece à Dr. ${ }^{a}$ Carla Nina, assistente de Medicina Geral e Familiar na USF Porta do Sol, pela orientação na elaboração deste trabalho.

\section{REFERÊNCIAS BIBLIOGRÁFICAS}

1. Murray CJ, Lopez AD. The Global Burden of Disease: a comprehensive assessment of mortality and disability from diseases, injuries, and risk factors in 1990 and projected to 2020. Harvard University Press; 1996.

2. Sambado C. Depressão afeta um em cada quatro portugueses. RTP. 2012 Sep 27. Available from: http://www.rtp.pt/noticias/index.php?arti$\mathrm{cle}=590570 \& \mathrm{tm}=8 \&$ layout $=121 \&$ visual $=49$

3. LUSA. Um em cada quatro portugueses já sofreu de depressão. Público. 2012 Sep 27. Secc Portugal, Sociedade. Available from: http://www. publico.pt/sociedade/noticia/um-em-cada-quatro-portugueses-ja-sofreu-de-depressao-1564773

4. Compton WM, Conway KP, Stinson FS, Grant BF. Changes in the prevalence of major depression and comorbid substance use disorders in the United States between 1991-1992 and 2001-2002. Am J Psychiatry. 2006;163(12):2141-7.

5. Gusmao RM, Xavier M, Heitor MJ, Bento A, Almeida JM. O peso das perturbações depressivas: aspectos epidemiológicos globais e necessidades de informação em Portugal [Depressive disorder burden: global epidemiological issues and information needs in Portugal]. Acta Med Port. 2005;18(2):129-46. Portuguese

6. American Psychiatric Association. Diagnostic and statistical manual of mental disorders. 4th revised ed. Washington, DC: APA; 2000. ISBN 9780890420256

7. Siwek J, Gourlay ML, Slawson DC, Shaughnessy AF. How to write an evidence-based clinical review article. Am Fam Physician. 2002;65(2):251-8.

8. Braga R, Melo M. Como fazer uma revisão baseada na evidência [How to make an evidence-based clinical review article]. Rev Port Clin Geral. 2009;25(6):660-6. Portuguese

9. Ebell MH, Siwek J, Weiss BD, Woolf $\mathrm{SH}$, Susman J, Ewigman B, et al. Strength of recommendation taxonomy (SORT): a patient-centered approach to grading evidence in the medical literature. Am Fam Physician. 2004;69(3):548-56.

10. Lam RW, Kennedy SH, Grigoriadis S, Mclntyre RS, Milev R, Ramasubbu R, et al. Canadian Network for Mood and Anxiety Treatments (CANMAT) clinical guidelines for the management of major depressive disorder in adults. III. Pharmacotherapy. J Affect Disord. 2009;117 Suppl 1:S26-43.

11. American Psychiatric Association. Practice guideline for the treatment of patients with major depressive disorder. 3rd ed. Arlington, VA:APA; 2010.

12. National Collaborating Centre for Mental Health. Depression: the NICE guideline on the treatment and management of depression in adults. Up- 
dated ed. London: The British Psychological Society and The Royal College of Psychiatrists; 2010. ISBN 9781904671855

13. Cipriani A, Koesters M, Furukawa TA, Nose M, Purgato M, Omori IM, et al. Duloxetine versus other anti-depressive agents for depression. Cochrane Database Syst Rev. 2012;10:CD006533.

14. Machado M, Einarson TR. Comparison of SSRIs and SNRIs in major depressive disorder: a meta-analysis of head-to-head randomized clinical trials. J Clin Pharm Ther. 2010;35(2):177-88.

15. Machado M, Iskedjian M, Ruiz I, Einarson TR. Remission, dropouts, and adverse drug reaction rates in major depressive disorder: a meta-analysis of head-to-head trials. Curr Med Res Opin. 2006;22(9):1825-37.

16. de Silva VA, Hanwella R. Efficacy and tolerability of venlafaxine versus specific serotonin reuptake inhibitors in treatment of major depressive disorder: a meta-analysis of published studies. Int Clin Psychopharmacol. 2012;27(1):8-16.

17. Gartlehner G, Hansen RA, Morgan LC, Thaler K, Lux L, Van Noord M, et al. Comparative benefits and harms of second-generation antidepressants for treating major depressive disorder: an updated meta-analysis. Ann Intern Med. 2011;155(11):772-85.

18. Bauer M, Tharmanathan P, Volz HP, Moeller HJ, Freemantle N. The effect of venlafaxine compared with other antidepressants and placebo in the treatment of major depression: a meta-analysis. Eur Arch Psychiatry Clin Neurosci. 2009 Apr;259(3):172-85.
19. Llorca PM, Fernandez JL. Escitalopram in the treatment of major depressive disorder: clinical efficacy, tolerability and cost-effectiveness vs. venlafaxine extended-release formulation. Int J Clin Pract. 2007Apr;61(4):702-10.

20. Cipriani A, Brambilla P, Furukawa T, Geddes J, Gregis M, Hotopf M, et al. Fluoxetine versus other types of pharmacotherapy for depression. Cochrane Database Syst Rev. 2005(4):CD004185.

21. Smith D, Dempster C, Glanville J, Freemantle N, Anderson I. Efficacy and tolerability of venlafaxine compared with selective serotonin reuptake inhibitors and other antidepressants: a meta-analysis. $\mathrm{Br} J$ Psychiatry. 2002May;180:396-404.

\section{CONFLITOS DE INTERESSE}

A autora declara não ter conflito de interesses.

\section{ENDEREÇO PARA CORRESPONDÊNCIA}

Ana Dias Amaral

Rua Rosária Martins Alves, 120

4510-652 Fânzeres

E-mail: ana.s.d.amaral@gmail.com

Recebido em 24-05-2013

Aceite para publicação em 12-05-2014

\section{ABSTRACT}

\section{A COMPARISON OF SNRI AND SSRI FOR INDUCTION OF REMISSION IN MAJOR DEPRESSIVE DISORDER: AN EVIDENCE-BASED REVIEW}

Aim: To evaluate the effectiveness of serotonin-norepinephrine reuptake inhibitors (SNRI) compared to selective serotonin reuptake inhibitors (SSRI) for induction of remission in major depressive disorder.

Data sources: PubMed, evidence-based medicine databases and Índex de Revistas Médicas Portuguesas.

Review methods: We searched guidelines, systematic reviews and meta-analyses published before $13^{\text {th }}$ January 2013 and randomized clinical trials published between $1^{\text {ts }}$ January 2011 and $13^{\text {th }}$ January 2013, using the MeSH terms depressive disorder, major; venlafaxine; duloxetine. Portuguese descriptors depressão and antidepressivos de segunda geração were also used. We used the Strength of Recommendation Taxonomy (SORT) from the American Family Physician for classifying the levels of evidence and the strength of recommendations.

Results: 223 articles were found. Three guidelines, nine systematic reviews and meta-analyses were selected. No RCT were found meeting the inclusion criteria. Both SNRI and SSRI are considered first-line therapeutic choices (level of evidence [LE] 1) and there is no clear evidence for the superiority of SNRI over SSRI (LE 2). There is consistent evidence for the higher efficacy of venlafaxine over fluoxetine and paroxetine (LE 2). Guidelines highlight the importance of choosing an antidepressant by considering tolerability and patient-related aspects (LE 1).

Conclusions: SNRI and SSRI are both evidence-based choices for induction of remission in depression (strength of recommendation [SOR] A). Drug and patient characteristics must be taken into account when choosing the antidepressant (SOR A). In a patient without co-morbidities, some SNRI show higher efficacy in remission of depression, but we cannot talk about a class effect. The choice of a SNRI over a SSRI to achieve remission of depression is not supported by the current evidence (SOR B).

Keywords: Major Depressive Disorder; Serotonin Uptake Inhibitors; Venlafaxine; Duloxetine; Remission Induction; Evidence-based Medicine. 